\title{
Magneto Hydro Dynamic two fluid flow of blood through stenosed artery
}

\author{
Surendra Kumar \\ University Institute of Engg \& Technology \\ Maharshi Dayanand University Rohtak- India \\ skymathmdu@rediffmail.com
}

\begin{abstract}
When blood flow through artery, the two-phase nature of blood as a suspension becomes important as the diameter of the red blood cell (RBC) becomes comparable to the tube diameter. The aim of the present study is to analyzed the effect of magnetic field on the plug flow region, shear stress in the core and plasma layer in two-fluid flow of blood through stenosed artery. Besides magnetic field, the effect of Womersley parameter, thickness of stenosis and width of plasma layer are also discussed. Generated data are analyzed and discussed through graphs.
\end{abstract}

\section{Keywords: Womersley parameter; Artery; Stenosis; plasma layer. \\ INTRODUCTION}

Quantitative understanding of the blood flow through arterioles is necessary for assessing the hemodynamic resistance and its regulation in the microcirculation as well as for analyzing mass transport processes. When blood flow through tubes, the two-phase nature of blood as a suspension becomes important as the diameter of the red blood cell (RBC) becomes comparable to the tube diameter. Womersley [20] studied the method for the calculation of velocity, rate of flow and viscous drag in arteries when the pressure gradient is known. Chaturani and Upadhya [3] studied a two fluid model for blood flow through small diameter tubes with the consideration as a couple stress fluid in the core and the plasma layer as a Newtonian fluid and observed that effective viscosity increases with tube radius. Srivastava and Srivastava [15] studied two-phase model of pulsatile blood flow with entrance effects. Sharan, and Popel, [13] studied a two-phase model for the flow of blood in narrow tubes. The model consists of a central core of suspended erythrocytes and a cell-free layer surrounding the core. It is assumed that the viscosity in the cell-free layer differs from that of plasma as a result of additional dissipation of energy near the wall caused by the red blood cell motion near the cell-free layer. Srivastava [16] studied particulate suspension blood flow through stenotic arteries, to analyze the effects of hematocrit and stenosis. Srivastava[16] studied a flow of a couple stress fluids representing blood through stenotic vessels with a peripheral layer and observed that the magnitudes of the flow characteristics increase with non-Newtonian behaviour of the core fluid, presence of a peripheral layer causes significant reduction in the magnitude of the flow resistance. The pulsatile flow of blood through a catheterized artery is analyzed by Sankar,[9], assuming the blood as a two-fluid model with the suspension of all the erythrocytes in the core region as a Casson fluid and the peripheral region of plasma as a Newtonian fluid. The estimates of the increase in the longitudinal impedance are considerably lower for the present two-fluid model than those of the single-fluid model. Shankar, and Lee, [10] analysed the pulsatile flow of blood through catheterized arteries by treating the blood as a two-fluid model with the suspension of all the erythrocytes in the core region as a nonNewtonian fluid and the plasma in the peripheral layer as a Newtonian fluid. The non-Newtonian fluid in the core region of the artery is represented by (i) Casson fluid and (ii) Herschel-Bulkley fluid. Kumar et al.[6] studied mathematical model of flow through a variable shape stenosed artery under the influence of magnetic field and discussed the effect of stenosis shape and maganetic field on the resistance to the flow. Sharma et al[12] worked on pulsatile unsteady flow of blood through porous medium. In their study the distribution of fatty cholesterol and artery-clogging blood clots in the lumen of the coronary artery is assumed as a porous medium. Sankar et al.[11] investigated Blood flow in the presence of a composite stenosis by examining the effects of red cell concentration, stenosis height and a peripheral layer on blood flow characteristics. A two-layered model with a particle-fluid suspension in the core region and a peripheral plasma layer without any particles is used to represent blood in there study. Srivastav [18] studied flow of blood through a narrow catheterized artery with axially non-symmetrical stenosis. In this study a two-layered model consisting of a core region of suspension of all the erythrocyte assumed to be a particle-fluid suspension and a peripheral layer of plasma.The aim of the present problem is to analyzed the effect of magnetic field on the plug flow region, shear stress in the core and plasma layer in two-fluid flow of blood through stenosed artery. Besides magnetic field, the effect of Womersley parameter, thickness of stenosis and width of plasma layer are also discussed.

\section{FORMULATION OF THE PROBLEM}

In the present study a long circular axisymmetric vessel is considered. The vessel is orienting in the $z$-direction. The radial and azimuthal co-ordinates are $r$ and $\theta$ respectively. The wall of the vessel is impermeable and due symmetric condition the non-vanishing component of flow velocity is axial velocity $w$ in the positive $z$-direction. The imposed magnetic field, $B_{0}$ is transverse to $z$-direction and of moderately small strength so that induced field be negligible. Under these considerations the flow in unidirectional, axisymmetric with no swirl in a circlular rigid vessel, its velocity can be taken as $\vec{q} \equiv \vec{q}(0,0, w(r, t))$. Thus, the fluid pressure is a function of the position $z$ and time $t$. The external magnetic field in applied transversally, so $\vec{B} \equiv \vec{B}\left(0,0, B_{0}\right)$.

The flow of the blood then described by the Navier-stokes equations and governing equation of motion by combining with Maxwell's equation. 


$$
\rho\left(\frac{\partial}{\partial t} \vec{q}+(\vec{q} \cdot \vec{\nabla}) \vec{q}\right)=-\vec{\nabla} p+\mu \Delta \vec{q}+\sigma(\vec{q} \times \vec{B}) \times \vec{B}
$$

$$
\rho \frac{\partial w^{*}}{\partial t^{*}}=-\frac{\partial p^{*}}{\partial z^{*}}-\frac{1}{r^{*}} \frac{\partial}{\partial r^{*}}\left(r^{*} \tau^{*}\right)-\sigma\left(B_{0}^{*}\right)^{2} w^{*}
$$

here astrics * denotes dimensional quantities.

The no-slip and axis-symmetric conditions provides the boundary conditions

$$
w^{*}=0 \text { at } r^{*}=h^{*}\left(z^{*}\right), \frac{\partial w^{*}}{\partial r^{*}}=0 \text { at } r^{*}=0
$$

where $h^{*}\left(z^{*}\right)$ defines the wall of the vessel which is a function of $z$ in the region of stenosis and equal to $R_{0}$ the radius of the vessel outside the stenosis region.

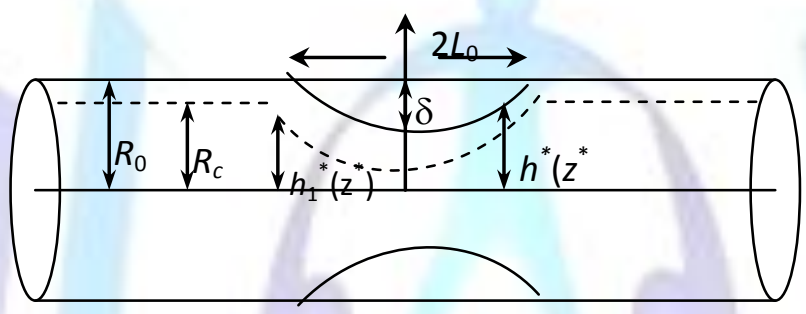

Fig. (1) Physical model of problem

The axis of the artery is coinciding with the z-axis. Considering the geometry of the stenosis as described

$$
h^{*}\left(z^{*}\right)=\left\{\begin{array}{ll}
R_{0}-\delta \cos \left(\frac{\pi z}{2 L_{0}}\right) & ;-L_{0}<z<L_{0} \\
R_{0} & ; \text { otherwise }
\end{array}\right\}
$$

where $R_{0}$ is the radius of artery in the region where stenosis is not present. $L_{0}$ is stenosis length.

The flow through a pipe using the relation

$$
\tau^{*}=-\mu \frac{\partial w^{*}}{\partial r} \text { and equation (2) is given by }
$$

$$
\begin{gathered}
\rho \frac{\partial}{\partial t^{*}} \int_{r^{*}}^{h^{*}} \frac{\tau^{*}}{\mu} d r^{*}=-\frac{\partial p^{*}}{\partial z^{*}}-\frac{1}{r^{*}} \frac{\partial}{\partial r^{*}}\left(r^{*} \tau^{*}\right)-\sigma B_{0}^{2} \int_{r^{*}}^{h^{*}} \frac{\tau^{*}}{\mu} d r^{*} \\
+\left(-\rho \frac{\partial}{\partial t^{*}}-\sigma B_{0}^{2}\right) w^{*}\left(h^{*}, t^{*}\right)
\end{gathered}
$$

The blood is taken as two layered fluid comparising with plasma in the peripheral region and casson fluid in the core region. Since in the plasma region the presence of $R B C$ are considered negligible and in the core region the $R B C$ are concentrated, therefore the effect of magnetic field will be only on the fluid that flowing in the core region. Using suffix $p$ and $c$ for plasma and core region respectively, then the governing equation of motion for core region and plasma region can be derived from (2.5) and given by as follows

For core region

$$
\rho \frac{\partial}{\partial t^{*}} \int_{r^{*}}^{h_{1}^{*}} \frac{\tau_{c}^{*}}{\mu} d r^{*}=-\frac{\partial p^{*}}{\partial z^{*}}-\frac{1}{r^{*}} \frac{\partial}{\partial r^{*}}\left(r^{*} \tau_{c}^{*}\right)
$$




$$
-\sigma B_{0}^{2} \int_{r^{*}}^{h_{1}^{*}} \frac{\tau_{c}^{*}}{\mu} d r^{*}+\phi\left(h_{1}^{*}, t^{*}\right)
$$

For plasma region

$$
\rho \frac{\partial}{\partial t^{*}} \int_{r^{*}}^{h^{*}} \frac{\tau_{p}^{*}}{\mu} d r^{*}=-\frac{\partial p^{*}}{\partial z^{*}}-\frac{1}{r^{*}} \frac{\partial}{\partial r^{*}}\left(r^{*} \tau_{p}^{*}\right)+\Phi\left(h^{*}, t^{*}\right)
$$

The corresponding boundary and interface conditions are

Symmetric condition

$$
\frac{\partial w_{c}^{*}}{\partial r^{*}}=0 \text { at } r^{*}=0
$$

Interface condition

$$
\left.\begin{array}{l}
w_{c}^{*}=w_{p}^{*} \\
\tau_{c}^{*}=\tau_{p}^{*}
\end{array}\right\} \text { at } r^{*}=h_{1}^{*}
$$

No-slip condition

$$
w_{p}^{*}=0 \text { at } r^{*}=h^{*}
$$

Here $\phi\left(h_{1}^{*}, t^{*}\right)$ and $\Phi\left(h^{*}, t^{*}\right)$ are function of $z^{*}$ and $t^{*}$ only defined by

$$
\phi^{*}\left(h_{1}^{*}, t^{*}\right)=\left(-\rho \frac{\partial}{\partial t^{*}}-\sigma B_{0}^{2}\right) w^{*}\left(h_{1}^{*}, t^{*}\right), \Phi^{*}\left(h^{*}, t^{*}\right)=-\rho \frac{\partial}{\partial t^{*}}\left[w^{*}\left(h^{*}, t^{*}\right)\right]
$$

\section{METHOD OF SOLUTION}

Introducing following non-dimensional quantities

$$
\begin{gathered}
\tau=\frac{\tau^{*}}{\left(P_{0} R_{c} / 2\right)}, \quad r=\frac{r^{*}}{R_{c}}, w=\frac{w^{*}}{\left(P_{0} R_{c}^{2} / 2 \mu\right)}, t=t^{*} \omega \\
-P_{0} p(t)=\frac{\partial p}{\partial z}=-P_{0}\left(1+\lambda e^{i \omega t}\right) \text { dimensional pressure gradient. } \\
M^{2}=\frac{\sigma B_{0}^{2} R_{c}^{2}}{\mu} \text { (Hartmann number), } \alpha=R_{c} \sqrt{\frac{\omega}{v}} \text { (Womersley parameter) }
\end{gathered}
$$

The pressure gradient in the vessel is taken pulsatile that in accordance of the nature of flow through the heart, $\lambda$ the amplitude of oscillation. Then the solution of governing equation of motion in dimensionless form in the core region and plasma region are given by :

$$
\tau_{p_{1}}=c_{1} I_{1}(\eta)+c_{2} K_{1}(\eta) \text { (Solution of modified Bessel's equation) }
$$

$$
\begin{gathered}
\tau_{c_{1}}=c_{1}^{\prime} I_{1}\left(r \sqrt{M^{2}+\alpha^{2} i \omega}\right), \quad \tau_{c_{0}}=\frac{2 I_{1}(M r)}{M I_{0}\left(h_{1} M\right)}, \tau_{p_{0}}=\frac{r}{2}+\frac{1}{r} \cdot h_{1}\left[\frac{2 I_{1}\left(M h_{1}\right)}{M I_{0}\left(M h_{1}\right)}-\frac{h_{1}}{2}\right] \\
w_{p_{1}}=c_{1}\left[I_{0}(\eta)-I_{0}\left(h \sqrt{i \omega \alpha^{2}}\right)\right]+c_{2}\left[K_{0}\left(\sqrt{i \omega \alpha^{2}} h\right)-K_{0}(\eta)\right], w_{c_{1}}=c_{1}^{\prime} I_{0}\left(r \sqrt{M^{2}+\alpha^{2} i \omega}\right)
\end{gathered}
$$

where

$$
\eta=\left(\sqrt{\alpha^{2} i \omega}\right) h_{1} \text { at the interface }
$$




$$
c_{1}=\frac{2 \lambda f_{2}}{f_{3} \sqrt{i \alpha^{2} \omega}}, \quad c_{2}=-\frac{2 \lambda f_{1}}{f_{3} \sqrt{i \alpha^{2} \omega}}, \quad c_{1}^{\prime}=\frac{2 \lambda\left(f_{2} I_{1}\left(h \sqrt{i \omega \alpha^{2}}\right)-f_{1} k_{1}\left(h \sqrt{i \omega \alpha^{2}}\right)\right.}{\sqrt{i \alpha^{2} \omega} f_{3} I_{1}\left(\sqrt{M^{2}+i \alpha^{2} \omega}\right)}
$$

And $f_{1}, f_{2}$ and $f_{3}$ are given by

$$
\begin{gathered}
f_{1}=I_{1}\left(h_{1} \sqrt{M^{2}+\alpha^{2} i \omega}\right)\left\{I_{0}\left(h_{1} \sqrt{\alpha^{2} i \omega}\right)-I_{0}(h \sqrt{i \omega \alpha})\right\} \\
-I_{0}\left(h_{1} \sqrt{M^{2}+\alpha^{2} i \omega}\right) I_{1}\left(h_{1} \sqrt{\alpha^{2} i \omega}\right) \\
f_{2}=I_{1}\left(h_{1} \sqrt{M^{2}+\alpha^{2} i \omega}\right)\left[K_{0}\left(h \sqrt{i \omega \alpha^{2}}\right)-K_{0}\left(h_{1} \sqrt{\alpha^{2} i \omega}\right)\right] \\
-I_{0}\left(h_{1} \sqrt{M^{2}+\alpha^{2} i \omega}\right) K_{1}\left(h_{1} \sqrt{i \alpha^{2} \omega}\right) \\
f_{3}=\left[f_{1} \cdot K_{0}\left(h \sqrt{i \omega \alpha^{2}}\right)+f_{2} I_{0}\left(h \sqrt{i \omega \alpha^{2}}\right)\right]
\end{gathered}
$$

Volume flow rate :

$$
Q=\pi \int_{0}^{h_{1}} r^{2}\left(\frac{d w}{d r}\right) d r+\int_{h_{1}}^{h} r^{2}\left(\frac{d w}{d r}\right) d r=\frac{\pi R^{3}}{\left(\tau_{c}\right)_{h_{1}}^{3}}\left[\left\{\tau_{c}^{4}+\theta \tau_{c}-\frac{2}{7} \sqrt{\theta} \cdot\left(\tau_{c}\right)^{\frac{7}{2}}\right\}_{h_{1}}-\frac{\theta^{4}}{28}-\theta^{2}\right]
$$

$\theta$ is dimension less yield stress.

\section{RESULTS AND DISCUSSION}

The data generated for the modeled problem are presented through figures to explain the effects of various physical parameters on the flow profiles. Figure 2 elaborate the effects of magnetic field on the square root of the velocity gradient (SRVG). It is plausible from the figure that with the increase of magnetic field the SRVG increase. Also, the plug flow region, the region where SRVG is zero, decreases with the increase of magnetic field. It is an interesting output which support the application of magnetic field on hemodynamics. Figure 3 represent the variation is SRVG versus radial distance at different values of thickness of the core region where blood cells are concentrated. The increase in core region does not effects the plug flow region but outside the plug flow region. SRVG increases with the increase of core region thickness. The increase in SRVG with the increase of core region thickness is reasonable which is caused due to the reduction is solid-fluid interfacial area per unit mass. In figure 4. Figure 5 and 6 we observe that the shear stress in core region as wall as in plasma layer increases with the increase of Hertmann member. But the variation in both the region are different in nature. In core region for small value of Hartmann number variation is linear but for higher value of Hartmann number linear variation not retained. While in the plasma layer, the variation in shear stress is linear. The physiological evidence that the low shear region is responsible for the atherosclerosis, the stenosis, in view of this, the applied magnetic field work in positive may to reduce the risk of atherosclerosis. In figure 7, the effect of plasma layer thickness on the shear stress in plasma layer is elaborated with the increase of plasma layer thickness the shear stress increases which is in favour to reduce the risk of atherosclerosis. The results are in good agreements with the results obtained by earlier researchers.

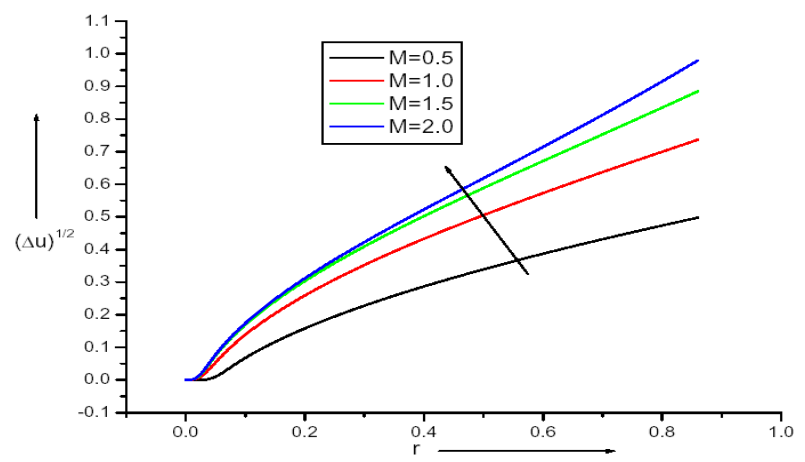

Fig. (2) Variation in square root of the velocity gradiant (SRVG) versus radial distance at different values of Hartmann number, $M($ at $\alpha=1.2, \omega t=\pi / 6)$ 


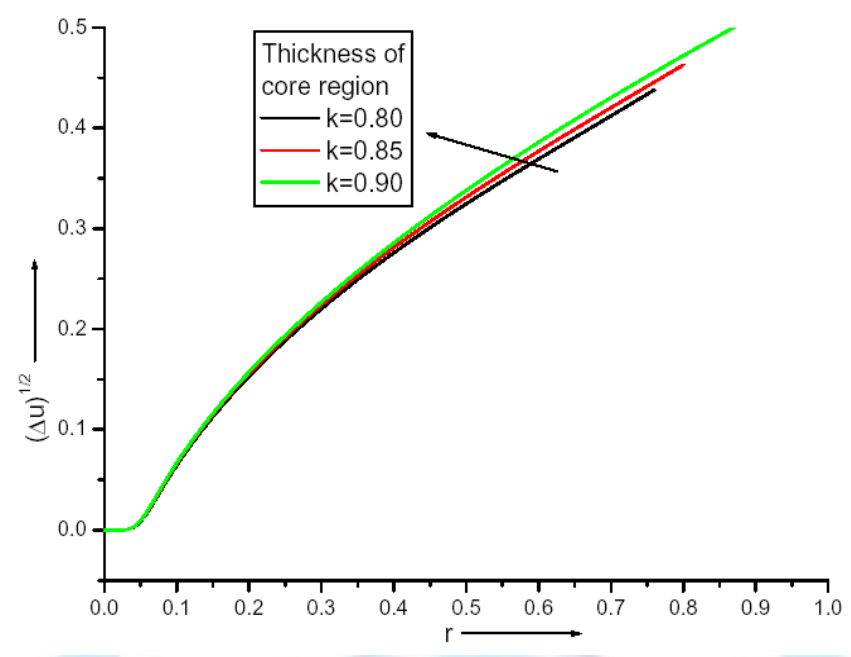

Fig. (3) Variation in SRVG versus radial radial distance at different values of thickness of the core region

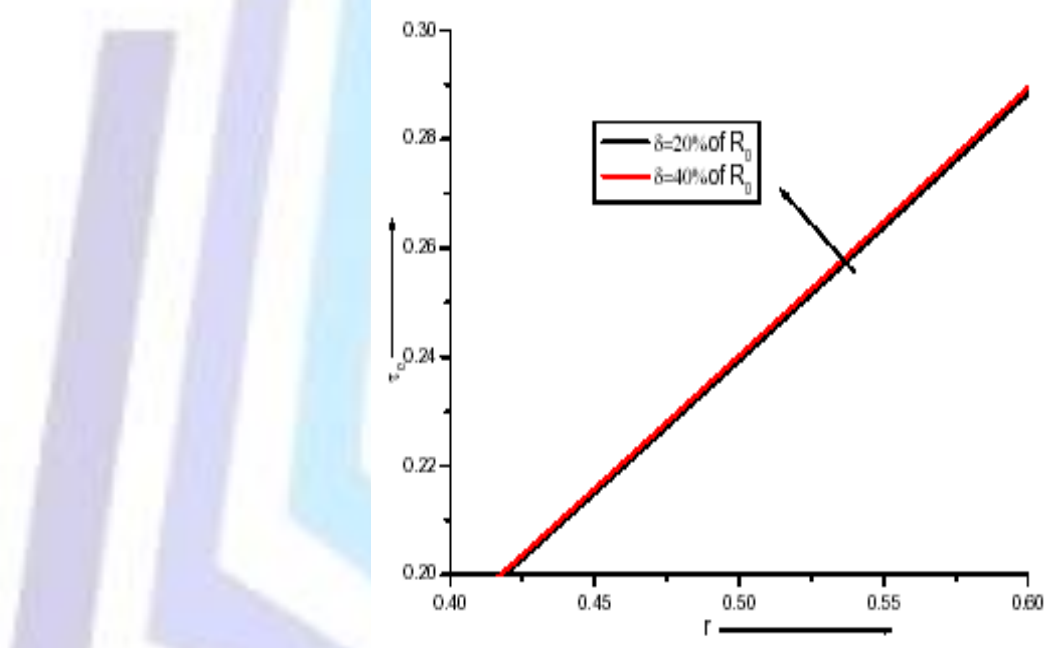

Fig. (4) Variation in shear stress in core region versus radial distance at different values of thickness of stenosis (at $M=0.5, \alpha=1.2, \omega t=\pi / 6)$

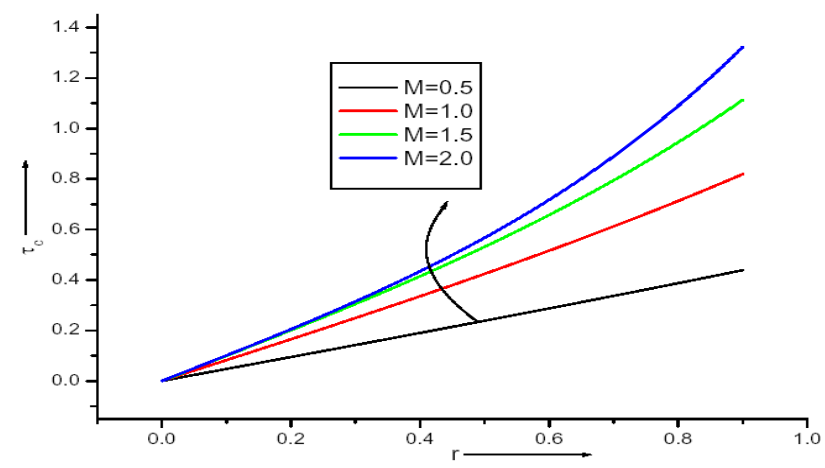

Fig. (5) Variation in shear stress in core region versus radial distance at different values of Hartmann number 
$M($ at $\alpha=1.2, \omega t=\pi / 6)$

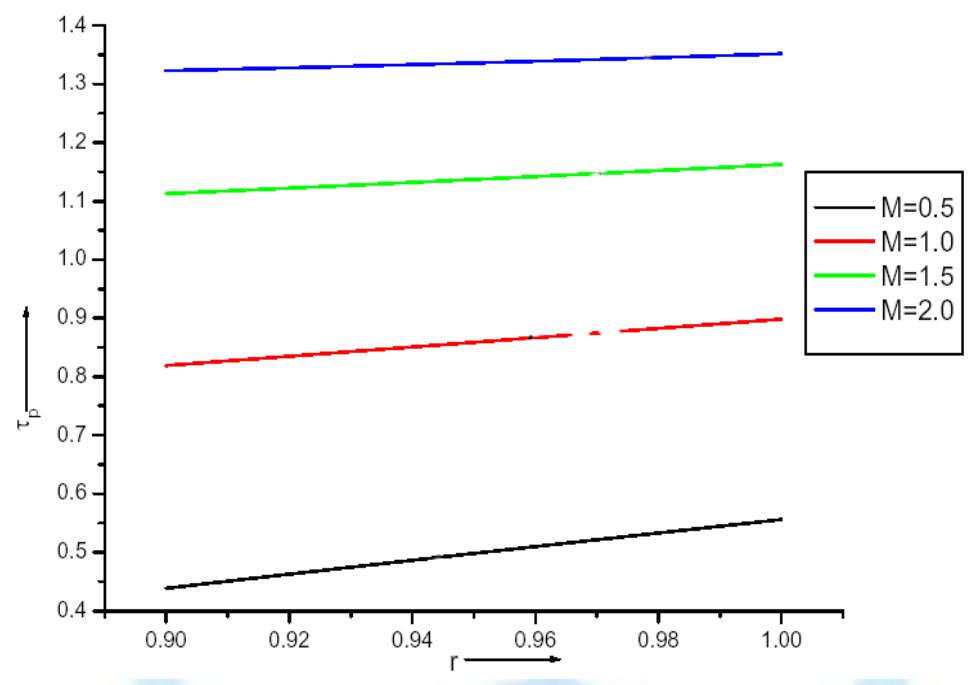

Fig. (6) Variation in shear stress versus radial distance at different values of Hartmann number $M$ (at $\alpha=1.2, \omega t=$ $\pi / 6)$

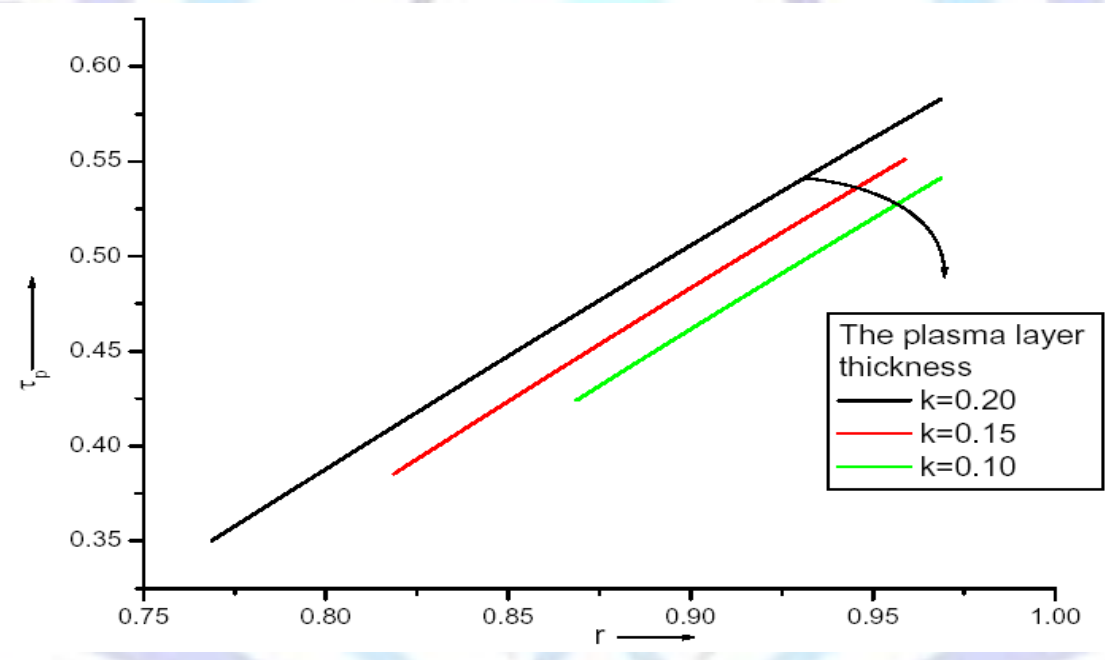

Fig. (7) Variation in shear stress versus radial distance at different values of thickness of plasma layer

\section{REFERENCES}

1. Adomian G.: Solving Frontier problems of Physics : The Decomposition Method, Kluwer Academic Publishers (1994).

2. Ahmad, Naseem and Misra, M.: Casson's fluid flow in an artery like elastic tube, Ind. Journal of Applied Sc. \& Computations, 10(1), 38- 46 (2003).

3. Chaturani, P. and Upadhya, V.S.: A two-fluid model for blood flow through small diameter tubes, Biorheology, Vol. 16, 109-118 (1979).

4. Fung, Y.C.: Biomechanics : Motion, Flow stress And growth, Springer Verlag, New York (1990).

5. Haldar, K.and Ghosh, S.N.: Effect of a magnetic field on blood flow through an indented tube in the presence of erythrocytes, Indian J. pure appl. Math, 25 (3), 345-352 (1994).

6. Kumar, S., Sharma M.K., Singh K. and Garg N. R.: MHD two phase blood flow through an artery with axially non- symmetric stenosis, International journal of Math. Sci. \& Engg. Appls., Vol. 5( II ), 6374 (2011).

7. Mazumdar J.N.: Biofluid Mechanics, World Scientific Publishing Co., Singapore (1992).

8. Mc Donald, D.A.: Blood flow in arteries, Edward Arnold(Publishers) Ltd., London (1974). 
9. Sankar, D.S.: A two-fluid model for pulsatile flow in catheterized blood vessels, international journal of Non-Linear Mechanics, 44, 337-351(2009).

10. Sankar, D.S. and Lee, U.: Pulsatile flow on two-fluid nonlinear models for blood flow through catheterized arteries a comparative study, Hindawi Publishing Corporation Mathematical Problems in Engineering, Art. 121757, 21(2010).

11. Sankar, A.R., Gunakala, S.R. and Comissiong, Donna M. G.: A Two-Layered Suspension Blood Flow Through a Composite Stenosis , Journal of Mathematics Research; 5( 4), 26-38 (2013).

12. Sapna, S.: Two-layered micropolar fluid flow through stenosed artery: Effect of peripheral layer thickness, International Journal of Physical Sciences, 4(11), 663-671(2009).

13. Sharan, M. and Popel, A.S.: A two-phase model for flow of blood in narrow tubes with increased effective viscosity near the wall, Biorheology, 38, 415-428 (2001).

14. Sharma, M.K., Bansal, K. and Bansal, S.: Pulsatile unsteady flow of blood through porous medium in a stenotic artery under the influence of transverse magnetic field, Korea-Australia Rheology Journal, 24(3), 181-189 (2012).

15. Srivastava, L.M. and Srivastava, V.P.: On two-phase model of pulsatile blood flow with entrance effects, Biorheology, 20, 761-777 (1983).

16. Srivastava, V. P.: Particulate suspension blood flow through stenotic arteries effects of hematocrit and stenosis shape, Indian J. pure appl. Math., 33 (9), 1353-1360 (2002).

17. Srivastava, V.P., Tandon, M. and Srivastav, R. K.: A Macroscopic Two-Phase Blood Flow through a Bell Shaped Stenosis in an Artery with Permeable Wall, Applications and Applied Mathematics: An International Journal, 7, 37-51(2012).

18. Srivastav, R.K.: Two-Layered Model of blood flow through arterial catheterization with non symmetric constriction, J. of Computation In Biosciences And Engineering , 2(2), 1-8 (2015).

19. Verma, N. and Parihar, R.S.: Effects of magneto-hydrodynamic and hematocrit on blood flow in an artery with multiple mild stenosis, International Journal of Applied Mathematics and Computation, 1(1), 30-46 (2009).

20. Womersley, J.R.: Method for the calculation of velocity, rate of flow and viscous drag in arteries when the pressure gradient is known, J.Physiol. 127, 553-563(1955).

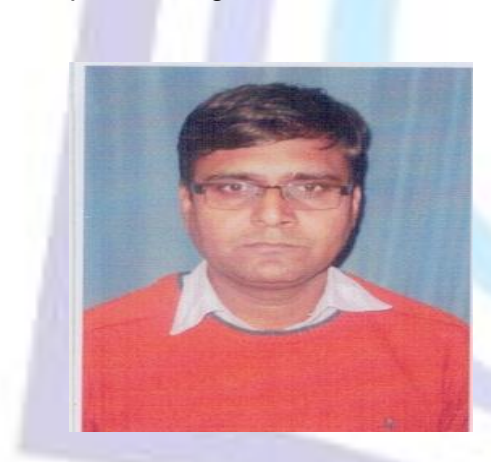

Surendra Kumar received the M.Sc degree in Mathematics from Maharshi Dayanand University Rohtak, Haryana (INDIA) in 2001 and Ph.D in Mathematics in 2011 from the same University. He is currently working in Department of applied Sciences at University Institute of Engineering \& Technology (M.D. University Rohtak, Haryana) India. His research interest includes Bio- Fluid Mechanics, Solid Mechanics and Fluid Mechanics. 